\title{
Angular Velocity, Magnetic Field, and Gauge Invariance
}

\section{Peng $\mathrm{Li}^{1,2}$ a , Xiao-Ping Qin ${ }^{1,2}$, Xue-Ping Cheng ${ }^{1,2}$ and Sheng-Wen $\mathrm{Qi}^{1,2}$}

${ }^{1}$ School of Mathematics, Physics and Information Science, Zhejiang Ocean University, Zhoushan 316022, China

${ }^{2}$ Key Laboratory of Oceanographic Big Data Mining \& Application of Zhejiang Province, Zhoushan 316022, China

apengli.physics@qq.com

Keywords: Pseudovector, Curl, Angular Velocity, Magnetic Field.

Abstract. We investigate two important applications of curl: the angular velocity and the magnetic field. The angular velocity is derived from the curl of the velocity field associated with the object, whereas the magnetic field is defined by the curl of a vector potential essentially determined by the current density. The gauge transformation of vector potential in electromagnetic theory is also discussed to complete the topic.

\section{Introduction}

In a preceding paper we have rigorously proved that the curl of a vector field is a pseudovector. Here we demonstrate the application of curl in physics by two examples: the angular velocity and the magnetic field. The angular velocity is first introduced via the conventional infinitesimal rotation operation, and then readdressed in an unusual approach via the curl of velocity field. Such treatment brings new insight into the nature of angular velocity. The conventional approach is better in treating mass point and rigid body dynamics whereas the present one is more suited for fluid mechanics or other field problems. The magnetic field is defined by the curl of a vector potential derived from the current source. Unlike the first example, the magnetic vector potential itself is not an observable, thus gauge arbitrariness arises and should be carefully handled. Historically, the vector potential itself does not occur in the Maxwell's equations. However, gauge invariance becomes a very important symmetry to be considered in modern field theories far beyond electromagnetism, especially in the understanding of interaction between fundamental particles.

\section{Stokes' Theorem}

Before diving into the applications we first look at a very important property of curl, as revealed by the Stokes' theorem

$$
\oint_{L} \boldsymbol{A} \cdot \boldsymbol{d l}=\int_{S} \nabla \times \boldsymbol{A} \cdot \boldsymbol{d} \boldsymbol{S} .
$$

It says that the circulation of $\boldsymbol{A}$ along an arbitrary closed trajectory $L$ can be calculated by the flux of its curl on the surface $S$ enclosed by $L$. Furthermore, if we shrink the trajectory $L$ and the surface $S$ into infinitesimal sizes, then it is easily seen that

$$
(\nabla \times \boldsymbol{A})_{n} \stackrel{S \rightarrow 0}{=} \frac{1}{S} \oint_{L} \boldsymbol{A} \cdot \boldsymbol{d l}
$$

Therefore, the normal component of curl can be concisely summarized as the circulation per area, which helps to understand the geometric essence of curl. The schematic view of Stokes' theorem is shown in Fig. 1. 


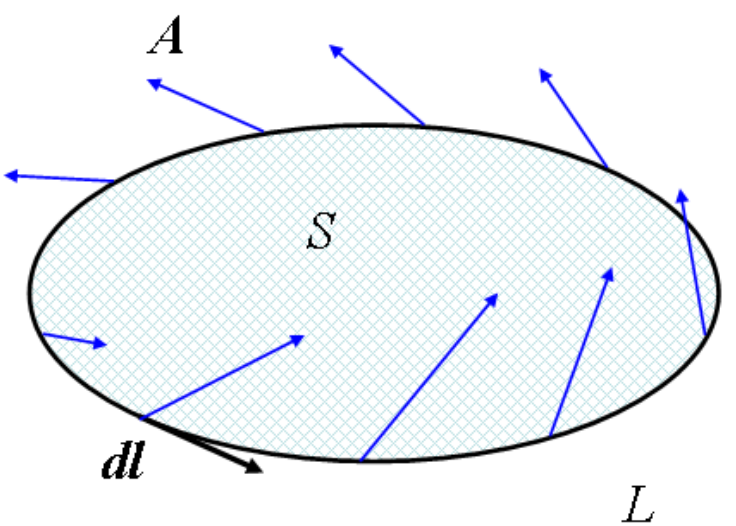

Fig. 1 The Stokes' theorem.

\section{Angular Velocity}

The angular velocity $\boldsymbol{\omega}$ is a vector describing the rapidity and the direction of rotational motion. It is originally defined via the infinitesimal rotation. First let us review the general rotation about an arbitrary axis $\boldsymbol{n}=\left(n_{x}, n_{y}, n_{z}\right)$, as summarized by the following transformation matrix

$$
\boldsymbol{A}^{\prime}=\boldsymbol{R}_{\boldsymbol{n}, \theta} \boldsymbol{A} \text {, where } \boldsymbol{R}_{\boldsymbol{n}, \theta}=I_{3}+\sin \theta \cdot \boldsymbol{N}+(1-\cos \theta) \cdot \boldsymbol{N}^{2} \text { and } \boldsymbol{N}=\left(\begin{array}{ccc}
0 & -n_{z} & n_{y} \\
n_{z} & 0 & -n_{x} \\
-n_{y} & n_{x} & 0
\end{array}\right) \text {. }
$$

(3)

In case of infinitesimal rotation the matrix becomes

$$
\boldsymbol{R}_{n, \Delta \theta}=I_{3}+\Delta \theta \cdot N,
$$

where the second-order infinitesimal is neglected. Clearly, the infinitesimal displacement of a vector $\boldsymbol{A}$ under such rotation reads

$$
\Delta \boldsymbol{A}=\boldsymbol{A}^{\prime}-\boldsymbol{A}=\left(\boldsymbol{R}_{\boldsymbol{n}, \Delta \theta}-I_{3}\right) \boldsymbol{A}=\Delta \theta\left(\begin{array}{ccc}
0 & -n_{z} & n_{y} \\
n_{z} & 0 & -n_{x} \\
-n_{y} & n_{x} & 0
\end{array}\right)\left(\begin{array}{l}
A_{x} \\
A_{y} \\
A_{z}
\end{array}\right)=\Delta \theta\left|\begin{array}{ccc}
\boldsymbol{e}_{\boldsymbol{x}} & \boldsymbol{e}_{\boldsymbol{y}} & \boldsymbol{e}_{z} \\
n_{x} & n_{y} & n_{z} \\
A_{x} & A_{y} & A_{z}
\end{array}\right| .
$$

The above equation can be rewritten as

$$
\frac{\Delta \boldsymbol{A}}{\Delta t}=\frac{\Delta \theta}{\Delta t}\left(n_{x}, n_{y}, n_{z}\right) \times\left(A_{x}, A_{y}, A_{z}\right) \equiv \boldsymbol{\omega} \times \boldsymbol{A} \text {, where } \boldsymbol{\omega} \equiv \frac{\Delta \theta}{\Delta t}\left(n_{x}, n_{y}, n_{z}\right) .
$$

(6)

Therefore, the rate of change of any vector $\boldsymbol{A}$ under rotation is obtained by a cross product with the angular velocity $\boldsymbol{\omega}$, which is a very useful vector quantity in describing the motion of rotational objects. We see that the direction of angular velocity is defined by the unit vector $\boldsymbol{n}$, which is the direction of the infinitesimal rotation, while the magnitude is defined by $\Delta \theta / \Delta t$, the rapidity of the rotation. However, it is not easy to tell that $\boldsymbol{\omega}$ is a pseudovector in this definition. 
Now let us consider the rotation of a rigid body, e.g., the disk rotating with angular velocity $\boldsymbol{\omega}$ normal to the disk as shown in Fig. 2. Assume the thread speed of its rim is $v$, and the radius is $r$, it is obvious that the magnitude of angular frequency is $\omega=v / r$. Interestingly, this can be alternatively calculated via equation (2). Divide the integral of velocity around the rim by the disk area we have

$$
(\nabla \times \boldsymbol{v})_{n} \stackrel{S \rightarrow 0}{=} \frac{1}{S} \oint_{L} \boldsymbol{v} \cdot \boldsymbol{d l} \stackrel{r \rightarrow 0}{=} \frac{1}{\pi r^{2}} v \cdot 2 \pi r=2 \frac{v}{r}=2 \omega .
$$

That is to say, the curl of the velocity field is exactly twice the angular frequency. This gives us a strong motivation to redefine the angular frequency as the curl of corresponding velocity field, i.e.,

$$
\omega \equiv \frac{1}{2} \nabla \times \boldsymbol{v} .
$$

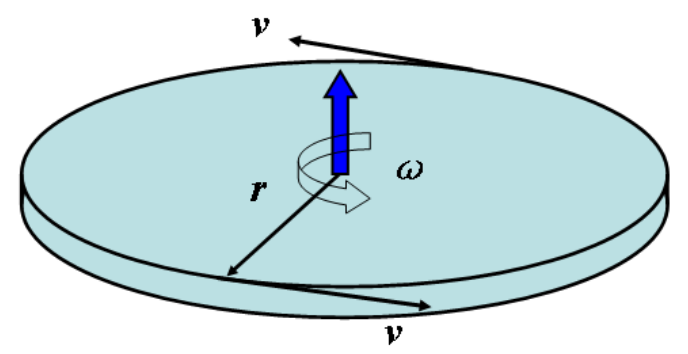

Fig. 2 The rotation of a rigid disk.

Then it is natural to say that angular velocity is a pseudovector because it is the curl of a real vector field $\boldsymbol{v}(\boldsymbol{r})$. Furthermore, this new definition leads to a completely different viewpoint on the nature of angular velocity. Instead of looking at a local point and investigate its rotation, now we study the global velocity field of a mechanical object and obtain the angular velocity as a derivative. An apparent benefit of this new approach is found in fluid mechanics, where vortices (whirls) are prevalent and differ from each other. Angular velocity can be used to describe the intensity of these vortices and can be easily calculated once the velocity field is obtained, either by measurement or by simulation. Additionally, it is also straightforward to verify that the angular velocity of a rigid body is identical everywhere, i.e., a rigid body can rotate with only one unique angular frequency.

\section{Magnetic Field}

In electromagnetic theory, the magnetic field is described by the curl of a vector potential, i.e.,

$$
B(r)=\nabla \times A(r) .
$$

In general, the vector potential is a function of the current density, which is the source of magnetic field. For a steady current source, the vector potential is independent of time and can be described by

$$
\boldsymbol{A}(\boldsymbol{r})=\frac{\mu_{0}}{4 \pi} \iiint_{V^{\prime}} \frac{\boldsymbol{j}\left(\boldsymbol{r}^{\prime}\right)}{\left|\boldsymbol{r}-\boldsymbol{r}^{\prime}\right|} d V^{\prime} .
$$

For time-varying sources, time lag between the source and the field locations due to finite speed of light has to be considered. In this case, the retarded vector potential reads [1] 


$$
A(\boldsymbol{r}, t)=\frac{\mu_{0}}{4 \pi} \iiint_{V^{\prime}} \frac{\boldsymbol{j}\left(\boldsymbol{r}^{\prime}, t-\frac{\left|\boldsymbol{r}-\boldsymbol{r}^{\prime}\right|}{c}\right)}{\left|\boldsymbol{r}-\boldsymbol{r}^{\prime}\right|} d V^{\prime} .
$$

Note this is an equation in accordance with the special relativity. The current density is derived from the motion of electrical charges as a product of charge density and local charge velocity, i.e.,

$$
\boldsymbol{j}(\boldsymbol{r})=\rho_{e}(\boldsymbol{r}) \boldsymbol{v}(\boldsymbol{r}) .
$$

Because velocity is a polar vector, now it is safe to say that the magnetic field $\boldsymbol{B}$ is a pseudovector based on equations (9-12).

Finally, it is worthful to spend a few lines on the gauge invariance of the magnetic field. Physically, the field vector $\boldsymbol{B}$ is the one that can be directly measured and is called the observable. However, the vector potential $\boldsymbol{A}$ is merely a theoretical tool to describe the field $\boldsymbol{B}$, as long as the equation (9) holds. In other words, the vector potentials in equation (10-11) are not unique for the corresponding magnetic field. This is true because of the equality

$$
\nabla \times(\nabla \psi) \equiv 0,
$$

where $\psi$ is an arbitrary scalar field. Therefore, we can perform a gauge transform such that

$$
\boldsymbol{A} \rightarrow \boldsymbol{A}^{\prime}=\boldsymbol{A}+\nabla \psi
$$

and find out that

$$
\boldsymbol{B}^{\prime}=\nabla \times \boldsymbol{A}^{\prime}=\nabla \times(\boldsymbol{A}+\nabla \psi)=\nabla \times \boldsymbol{A}+\nabla \times(\nabla \psi)=\nabla \times \boldsymbol{A}=\boldsymbol{B} .
$$

Obviously, the gauge transformation [equation (14)] of vector potential does not change the magnetic field $\boldsymbol{B}$. This kind of arbitrariness in vector potential brings an additional problem: the choice of gauge. In other words, although the curl $\nabla \times \boldsymbol{A}$ is determined by the observable field $\boldsymbol{B}$, the divergence $\nabla \cdot \boldsymbol{A}$ is still undetermined because no restriction has been made. Mathematically, to determine a unique solution of $\boldsymbol{A}$ we must not only specify the curl but also the divergence. The theoretical choice made to specify the divergence of vector potential is called the choice of gauge. For example, the equation (10) is actually obtained under the assumption $\nabla \cdot \boldsymbol{A}=0$, i.e., the Coulomb gauge. An illustration of the vector potential of a toroidal inductor under the Coulomb gauge is shown in Fig. 3. Although Coulomb gauge is convenient in treating steady magnetic fields, the Lorenz gauge is more suited for dynamic problems such as electromagnetic radiation. 


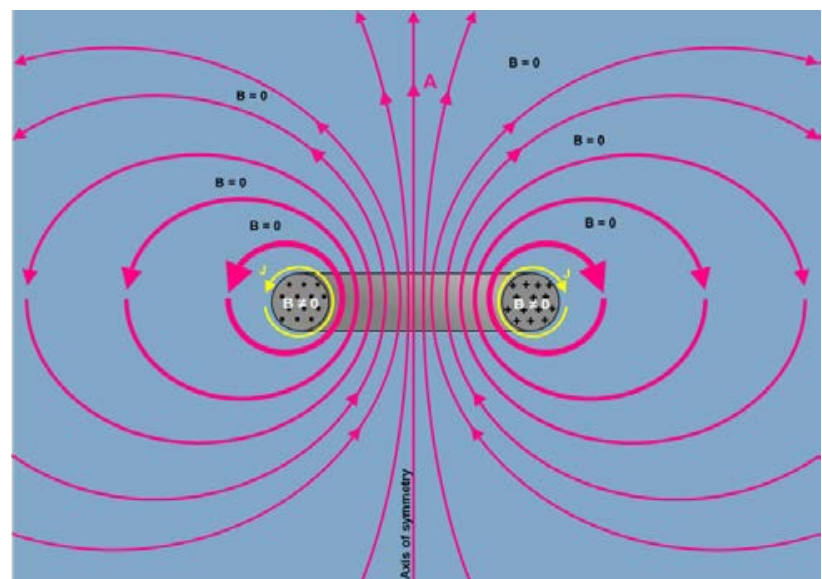

Fig. 3 The vector potential $\boldsymbol{A}$ around a toroidal [2].

\section{Acknowledgement}

This work was supported by the Teaching Enhancement Program of Zhejiang Ocean University and NSFC Grant No. 11505154, 11305141, 11104248, and 11204271.

\section{References}

[1]. J. D. Jackson, Classical Electrodynamics, John Wiley \& Sons, 1999.

[2]. Information on: https://en.wikipedia.org/wiki/Magnetic_potential 